\title{
Podocyturia: What is in a name?
}

\author{
Hernán Trimarchi \\ Nephrology and Kidney Transplant Unit, Department of Medicine, Hospital Británico de Buenos Aires, \\ Buenos Aires, Argentina
}

\begin{abstract}
The podocyte is a highly differentiated cell located in the outer space of the glomerular basement membrane that deals with many different functions. This phylogenetically preserved cell that is responsible for the virtually absence of proteins in the urine lacks of the capacity to divide under normal conditions. When podocytes receive molecular insults, which normally occur during stress conditions as glomerulonephritis, hyperfiltration or metabolic disturbances, they adapt to the new situation by contracting their actin fibers. This adaptive behavior puts at risk the quality of the plasmatic filtration due to the denudation of the glomerular basement membrane, the potential mesangial inflammation and the appearance of proteinuria; podocytes run the risk of detachment from the basement membrane due to a decrease in the adherence to the surrounding matrix after contraction, a process called foot processes effacement. Podocytes change their shape and under constant mechanical stress they finally detach, rendering the glomerular basement membrane unprotected unless other contiguous podocytes are capable of covering the surface. However, these still anchored podocytes are generally also under the same stress situation and follow the same pathway. Podocyturia refers to the presence of these differentiated cells in the urinary sediment. Noteworthy, the podocytes that are encountered in the urine are viable despite the glomerular hostile environment and the urinary acidity. Podocyturia can precede proteinuria and can aggravate it. Therefore, in diseases that can threaten the glomerular normal environment, the presence and the quantification of urinary podocytes can be of remarkable relevance, as it can herald or accompany the appearance of proteinuria, and could offer another view to the interpretation and clinical approach and outcome of proteinuria. However, its identification needs a wide-spread training among biochemists and technicians, as well as commercially available kits.
\end{abstract}

Key words: Glomerular basement membrane, nephron, podocyte, podocyturia, proteinuria

\section{THE NORMAL PODOCYTE}

The podocyte is located in the outer side of the glomerular basement membrane and consists of a large cell body facing the urinary space. It is attached to the underlying glomerular basement membrane (GBM) with long interdigitating foot processes embracing the capillary loop covering the GBM. Integrins (predominantly $\alpha 3 \beta 1$ integrin) and $\alpha$ - and $\beta$-dystroglycans bind podocytes to the GBM. Between the foot processes, the filtration slit is bridged by the slit diaphragm, a structure designed to permit the passage of water and small solutes as electrolytes; however, the small size of the pores, which measure around $10 \mathrm{~nm}$, limits the passage of larger proteins including albumin. Moreover, the negative charge of the cellular glycocalyx and of the GBM impedes the filtration of negative charged molecules as albumin itself. The slit diaphragm is connected to the actin cytoskeleton located in the cytoplasm of podocytes. Signaling pathways from these slit diaphragm molecules regulate actin and allow podocytes to actively alter their shape through the interaction with other proteins as synaptopodin. The mechanical stability of podocytes may be further strengthened during injury by the up-regulation of intermediate filament proteins such as nestin and vimentin. Genetic or acquired abnormalities in slit diaphragm components and/or in podocyte protein cytoskeleton may result in marked proteinuria. ${ }^{[1,2]}$

The most important functions of podocytes include: structural support of the capillary loop, due to the podocyte possession of 
an extensive and dynamic actin cytoskeleton, allowing contraction and support of the glomerular capillary cells, counteracting the glomerular capillary hydrostatic pressure; the podocyte intervenes in the prevention of proteinuria; ${ }^{[3,4]}$ the podocyte maintains the glomerular filtration barrier by removing protein and immunoglobulins that may clog the filter; podocytes participate in the synthesis and possible repair of the GBM, as type IV collagen; podocytes interact with glomerular endothelial cells and mesangial cells to ensure their normal function. The clearest example is the production of VEGF (vascular endothelial growth factor) by the podocyte, which traverses the GBM counter to the flow of glomerular filtration, to act on VEGF receptors on the endothelial cell to keep a healthy fenestrated endothelium, or the synthesis of PDGF (platelet-derived growth factor), produced during development by podocytes to induce the genesis of mesangial cells; podocytes may be a component of the innate immune system, possibly playing a surveillance role for pathogens or abnormal proteins in Bowman space. ${ }^{[5]}$ They constitutively express Toll-like receptor 4 which upon signaling stimulates chemokine production. ${ }^{[6]}$ Furthermore, lipopolysaccharide, a component of cell wall of Gramnegative bacteria, triggers synthesis of $\mathrm{B} 7-1$ or $\mathrm{CD} 80$, which (in addition to $\mathrm{T}$ cell co-stimulation) leads the actin cytoskeleton and slit diaphragm proteins to reorganize, resulting in the development of proteinuria. ${ }^{[1,7,8]}$

\section{PODOCYTE AND CELL DIVISION}

In the absence of disease, nephron number at birth in mammals largely exceeds the needs, enabling the increase of body mass related to childhood growth until adult age. ${ }^{[1,9,11]}$ However, the number of nephrons declines by about $50 \%$ after the age of 60 years, which may explain the reason why an inadequate number of nephrons turns up becoming an important determinant of renal failure in humans once life expectancy increased beyond evolutionary requirements (the aging process). ${ }^{[9-11]}$ However, despite an adequate number of nephrons, a silent and constant podocyte loss can lead to glomerulosclerosis. This can occur at a sustainable pace, when the normal decline of renal function accompanies aging in subjects with a reduced number of nephrons at birth (premature birth), or at an accelerated fashion, as it occurs in primary or secondary glomerulonephropathies. In general, the inability of podocytes to proliferate is a main cause of chronic kidney disease and kidney failure. ${ }^{[9-12]}$

During development, renal progenitors can act as precursors to all renal epithelial cells of the cortical nephron, being able to generate the glomerular or the tubular epithelial cell lineages. ${ }^{[13-18]}$ In general, the capacity of mammals to generate new nephrons ceases at the time of birth. Later, in adult kidneys, bipotent renal progenitors that display the capacity to differentiate into glomerular or tubular epithelial cells localize at the urinary pole, which represents the junction between the tubular and the glomerular epithelium (the proximal convoluted tubule and Bowman's capsule).$^{[16]}$ In the adult mammalian nephron, renal stem and progenitor cells are encountered in a cluster localized at the urinary pole and within the Bowman's capsule and as scattered cells within the tubular compartment of the nephron. Podocyte-committed progenitors, which display transitional features of progenitors and podocyte cells, localize along the Bowman's capsule and can only differentiate into a podocyte. ${ }^{[17]}$ However, an important controversy exists with respect to this statement. No proven in vivo documentation exists that can support a programmed migration of progenitor cells located in Bowman's capsule to denuded areas of the GBM as podocytes detach, either under normal or pathologic conditions. Finally, due to its location against a constant flow of filtration, it appears difficult for podocytes to enter cell division at the GBM. This situation may explain why the nephron and consequently podocyte number at birth far exceeds the needs of a lifetime. ${ }^{[11]}$

\section{PODOCYTE DAMAGE AND PODOCYTURIA}

The classic clinical feature of podocyte injury is proteinuria. Due to its location in the GBM and to the constant exposure to a fluid flow, podocytes are constantly exposed to the risk of detachment. The unique situation of podocytes as floating cells, adhering to the GBM only by their foot processes and being exposed to shear stress from the flow of filtrate, makes podocytes susceptible to detachment. ${ }^{[19]}$ Injury to mesangial or endothelial cells, which are in direct contact with the blood, typically leads to inflammatory kidney disease, named glomerulonephritis. By contrast, as podocytes sit on the outside of the GBM, and are excluded from the circulation by the GBM, injury usually does not lead to leukocyte recruitment and inflammation, but rather disrupts the glomerular filtration barrier, leading to a morphologic non-inflammatory histologic pattern and proteinuria, despite an important cytokine local involvement. ${ }^{[20]}$ This may be due to an impaired slit diaphragm integrity or possibly to alterations in podocyte charge or shape, namely foot process effacement (FPE). FPE is an active process that occurs due to actin cytoskeleton rearrangement. Recent evidence links activation of the podocyte integrin, $\alpha V \beta 3$, by molecules such as the urokinase receptor to the development of FPE and potentially the promotion of cell motility. ${ }^{[21,22]}$ The podocyte response to injury and resulting mechanisms of proteinuria are well described in recent reviews and should be viewed as an adaptation to injury in order to avoid detachment. ${ }^{[4,19,23]}$ According to Shankland, 
podocytes are terminally differentiated epithelial cells that appear to attempt to maintain this quiescent differentiated phenotype at all costs, even at the expense of developing glomerulosclerosis, which, if balanced and limited, can be viable. ${ }^{[24]}$

In this regard, podocyte foot processes contraction and effacement must be viewed as an adaptive response to injury in order to prevent podocyte loss. As a consequence, slit diaphragms are widened and protein filtration is facilitated. ${ }^{[19-24]}$

However, podocyte loss has also been reported to occur in normal conditions. In the adult human kidney, there are roughly 500 podocytes per glomerular tuft, and their rate of turnover is very slow. Considering the fact that there exist 2 million glomeruli in both kidneys, approximately 1 billion podocytes populate both kidneys. In addition, it has been calculated that around 400 podocytes are lost in the urine every day. Finally, it has been demonstrated that when a glomerulus loses between $20 \%$ and $40 \%$ of its podocyte content (around 100-200 podocytes), it renders itself to sclerosis and obliteration. ${ }^{[12,25]}$ Taken together, this means that 146,000 podocytes are lost in 1 year, and 1,460,000 million would be lost in a decade. This is supposed to occur in normal conditions, and several co-morbidities could of course augment this rate loss. According to histologic observations, this podocyte loss could be uneven as to the distribution of glomerular structures, being more frequently found at the sub-capsular area, where obliterated glomeruli are encountered in kidney tissue of non-glomerular diseases. Moreover, if the loss of 100-200 podocytes can lead to one obliterated glomerulus, in 1 year around 1460 glomeruli would be lost, and 14,600 in 10 years time, again in normal conditions. However, in the general aged population this intriguing reality that shows a continuous physiological loss of this non-substitutable cell is not accompanied by important proteinuria. On the other hand, renal function decline is the rule in the normal aging process. This could be due to the expansion of neighboring podocytes covering the denuded GBM and tempering the degree of albumin filtration. Another provoking explanation could be - as mentioned above - the replacement of these podocytes by local pluripotential cells located in the parietal compartment of Bowman's capsule. Some parietal epithelial cells express stem cell markers as CD24 and CD133, and may act as renal progenitor cells. As they approach the vascular pole, they gradually lose these stem cell markers, acquire podocyte specific markers, and may migrate onto the GBM. ${ }^{[15]}$ The ability of the podocyte to migrate within the glomerular tuft has been elegantly demonstrated using time lapse fluorescence microscopy. ${ }^{[2]}$ However, not all replacement podocytes are derived from resident renal cells. Transplant studies in which male recipients received female kidney allografts have demonstrated the presence of podocytes derived from the recipient male in approximately $50 \%$ of cells, suggesting a bone marrow derived stem cell source. ${ }^{[27]}$ Moreover, parietal epithelial cells express podocyte markers in several experimental models of glomerulonephritis. ${ }^{[28]}$ This phenotype switching may also portrend adverse consequences for the glomerulus, possibly contributing to the development of adhesions and scars between the glomerular tuft and Bowman's basement membrane. Although some in vitro reports have identified these cells, no proven in vivo documentation has been published yet. If there is a lack of coverage, ballooning of the capillary loop ensues, synechial attachment of denuded areas to Bowman's capsule follows and focal and segmental glomerulosclerosis develops. ${ }^{[19,29]}$

\section{THE IDENTIFICATION OF PODOCYTES}

Hara et al. ${ }^{[30-33]}$ have described podocalyxin-positive cells in urinary sediments of patients with glomerular diseases. They showed that changes in semi-quantitative measures of podocyturia seem to correlate with disease activity as assessed by biopsy and to decline with treatment, ${ }^{[32,33]}$ suggesting that urinary shedding of podocytes may represent a real-time measure of podocyte loss from the glomerulus. However, podocytes are shed due to necrosis or to apoptosis in situ, or they can still be viable when they detach from the GBM. ${ }^{[30-34]}$ At preliminary observations, we have recently identified urinary podocytes both in health and glomerular diseases employing synaptopodin, a post-mitotic protein, as the cellular marker (ongoing study). Synaptopodin may suggest that some maturation exists in the viable podocytes. ${ }^{[24,35]}$

Podocytes can be identified by the expression of specific markers as podocalyxin, WT1, synaptopodin, podocin, nestin among others. With the use of a cytospin method to quantitate podocyte excretion, ${ }^{[30]}$ and normalizing podocyte number by the urinary creatinine content, the number of podocytes shed by healthy controls and patients with inactive disease appears to be lower than in patients with active glomerular disease. The rate of podocyte excretion appears to reflect both disease activity and disease type. Quantitative podocyturia tend to be greater in patients with histologic proliferative patterns as active lupus nephritis than in those with less inflammatory patterns. ${ }^{[12,36]}$

\section{PODOCYTURIA, APOPTOSIS AND DETACHED CELL VIABILITY}

Since podocytes adhere to the GBM by multiple cell processes, detachment of a podocyte proceeds through 
stages, in which some foot processes lose their connections while others still are affixed to the GBM. Such focal detachments do not necessarily indicate that a podocyte as a whole has detached or has entered an irreversible process of disconnection from the GBM. Partial detachments are likely reversible to a certain degree. Podocytes in the process of detachment regularly show remarkable shape changes, with FPE being frequently present. The vast majority of these cells appear to be viable with normal shape and organelle distribution and appearance. When a podocyte undergoes detachment, contiguous podocytes establish contacts to each other, forming a new interconnected net. According to Kriz, binucleate cells can be present, corroborating previous observations that urinary podocytes frequently have two cell nuclei. ${ }^{[12,19,37]}$ Detachment is mostly encountered in podocytes with effaced foot processes. FPE frequently appears to precede detachment. A special type of detachment affects podocytes that come to lie at the opening of Bowman's capsule to the proximal tubule. This is the region of the highest flow velocities of filtrate. Such podocytes are in extreme danger of being lost by detachment. Of note, in contrast to podocytes elsewhere in these glomeruli, only podocytes protruding into the urinary orifice have been found to exhibit FPE. Thus, also in these cases, FPE appears to be a reaction to mechanical stress and to precede detachment. Podocytes encountered in the process of detachment in transmission electron micrograph (TEM) images of experimental models appear viable and display FPE. Thus, FPE appears to precede detachment. The elaborate organization of the cytoskeleton associated with FPE contradicts the interpretation that FPE simply represents a form of cell injury. FPE is reversible, even when it is extensive. Thus, FPE seems to represent a specific, reactive phenotype of the podocyte, which may enhance adhesion to the GBM and may limit the risk of detachment, at least for some time. Consequently, FPE can be viewed as a strategy for podocytes to avoid detachment, providing the basis of the possibility for a reconstitution of the filtration barrier when local conditions have improved. FPE is clearly not always successful, as indicated by the widespread presence of urinary podocytes in many diseases. ${ }^{[19]}$

Podocyturia also appears to be greater in active than in inactive stages of the same disease. Moreover, proteinuria appears not to be correlated with the degree of podocyturia, while others have found the opposite. ${ }^{[12,38]}$ The hypothesis that podocytes encountered in the urine are not all undergoing apoptosis has been a question of debate. According to Vogelman et al., apoptosis is present in about one-half of the urinary podocytes to be examined. ${ }^{[12]}$ Apoptosis of glomerular podocytes in situ has also been described in animal models of progressive glomerular sclerosis. ${ }^{[31]}$ On the other hand, Kriz et al. have been suggesting that there are some pitfalls and controversies about this statement. ${ }^{[19]}$ When urinary samples are examined employing terminal uridine deoxynucleotidyl transferasemediated dUTP nick-end labeling (TUNEL) staining, the apoptotic count of podocytes appears to be higher due to the lack of specificity of the method. ${ }^{[39]}$ In advanced stages of glomerular diseases, Kriz et al. have used TEM (a gold standard to demonstrate apoptosis) to assess podocytes in the urine and were unable to find apoptotic cells. They remark that the spillage of lysosomal enzymes into the podocyte cytoplasm may represent the proximate cause for an in situ cell death. ${ }^{[23]}$ Apoptosis may have occurred as a consequence of having lost a connection to a matrix substrate during their passage through the nephron. Moreover, as remarked by Vogelman et al., it cannot be ruled out that in subjects with glomerular disease podocytes may detach from the glomerular tuft intact and viable and a subsequent exposure to extreme conditions in the urine then induces them to undergo apoptosis. ${ }^{[12]}$ Interestingly, podocyte viability is not influenced by extreme conditions as acid urinary $\mathrm{pH}$. Urinary podocyte viability has also been confirmed by their ability to grow in culture. ${ }^{[40,41]}$ Podocytes cultured from the urine of patients with active glomerular disease show the same morphology and growth pattern as outgrowths from glomeruli isolated from whole kidney, while those cultured from healthy subjects have less growth capability and die sooner. These results suggest that in glomerular diseases podocytes are shed from the glomerulus not as a result of a defect in the podocytes themselves, but rather as a response to some damaging but sublethal stimulus in the glomerular compartment. For example, activated complement or even immunocomplexes may disrupt integrin- or dystroglycanmediated podocyte adhesions, ${ }^{[42]}$ whereas exposure to a circulating factor as suPAR in primary FSGS might disrupt the actin cytoskeleton. ${ }^{[43]}$ Podocytes from normal healthy individuals, on the other hand, may be shed principally when they are senescent, resulting in their limited potential for replication in culture. ${ }^{[12]}$

The behavior of podocytes under in vivo conditions is different from those collected from the urine and grown under in vitro conditions thereafter. In vitro podocytes are not fully differentiated despite they express podocyte-specific proteins. In addition, they undergo significant cell division, something completely uncharacteristic of in situ/in vivo podocytes. ${ }^{[23]}$ This finding may have important implications, as conclusions drawn from in vitro studies may be unreliable when extrapolated to the in situ/in vivo scenario.

Finally, those recovered alive podocytes are capable of growing in culture, albeit for a limited number of cycles; this phenomenon has been documented in many primary or secondary glomerulopathies, as focal 
and segmental glomerulosclerosis, IgA nephropathy, active lupus nephritis, hypertension, rapidly progressive glomerulonephritis. ${ }^{[1,25,36]}$

As in either health and disease, urinary podocytes show up as round viable cells with a demonstrated capacity to proliferate in vitro. ${ }^{[12]}$ Maestroni et al. have recently investigated whether an underlying process of dedifferentiation existed in this phenomenon of podocyte detachment. ${ }^{[44-46]}$ They have reported that in healthy subjects viable podocyturia consisted of dedifferentiating cells coexpressing mature podocyte antigens plus immaturity markers. ${ }^{[4]}$ The presence of immature podocytes could be interpreted as an ongoing dedifferentiation process allowing a still unknown stem cell pool to replace dying or detaching podocytes, probably from Bowman's capsule. Although this hypothesis cannot be excluded, it has not been shown under in vivo conditions. Maestroni et al. have also demonstrated that no niches of undifferentiated stem cells expressing immature transcription factors in the absence of podocyte markers could be detected inside the glomerulus. Moreover, according to the markers employed in vivo and in vitro, they have shown that in healthy individuals, the process of podocyte dedifferentiation precedes detachment from the basement membrane for clusters of immature podocytes were detected inside normal glomerular sections. ${ }^{[4]}$ However, it does not exclude that podocytes may also detach when still differentiated.

\section{PODOCYTURIA, PROTEINURIA AND OTHER CLINICAL IMPLICATIONS}

Notwithstanding the cause, podocytopenia appears to correlate with disease progression..$^{[1,47,48]}$ Due to the above-mentioned degree of podocyte loss conditioning glomerular viability, it is important to remark the fact that although proteinuria is a relevant marker of disease progression and cardiovascular disease, its occurrence is denoting a late or advanced state of glomerular damage. Podocyturia occurs even when proteinuria is absent or only detected at microalbuminuric stages. Moreover, the discrepancy that podocyturia may or may not correlate with proteinuria may be due to the stage at which the phenomenon is assessed, and also to the glomerular disease under consideration. Glomerular diseases display different degrees of podocyturia. ${ }^{[36]}$ At early stages, only podocyturia may be encountered. As the glomerular disease progresses, both podocyturia and proteinuria may be nicely correlated. At more advanced and terminal states, podocyturia may decline and proteinuria could still be elevated, and again both variables may not be correlated. Finally, in endstage renal disease, both podocyturia and proteinuria quantifications have not been reported. However, these are merely unproven conjectures that need to be studied further. In this setting, we have previously reported the existence of proteinuria in chronic hemodialysis patients and its correlation with inflammatory and cardiovascular markers. ${ }^{[4]}$ In our yet unpublished data about podocyturia and proteinuria in chronic kidney disease at stage 5 due to glomerulonephritis, we have found the concomitant occurrence of proteinuria and podocyturia. However, there appears to be no correlation between both variables at this stage of kidney disease.

\section{CONCLUSIONS}

Podocyturia is a physiologic event that can hasten glomerulosclerosis in the setting to a glomerular insult, mainly due to the inability of podocytes to divide under in vivo conditions. Under normal conditions, daily podocyte detachment does not cause proteinuria because the pool of podocytes outnumbers the needs of a glomerulus during a lifespan, and the denuded GBM is covered by contiguous podocytes. When podocyturia exceeds its normal range, proteinuria appears. The diagnosis of proteinuria is therefore a late event. Early urinary markers are mandatory to be found to support an adequate in-time treatment. Podocyturia could be employed at many instances: as an early marker of glomerular damage, for follow-up and after the prescription of therapy.

\section{REFERENCES}

1. Jefferson JA, Alpers CE, Shankland SJ. Podocyte Biology for the Bedside. Am J Kidney Dis 2011;58:835-45.

2. Jefferson JA, Nelson PJ, Najafian B, Shankland SJ. Podocyte Disorders: Core Curriculum 2011. Am J Kidney Dis 2011;58:666-77.

3. Haraldsson B, Jeansson M. Glomerular filtration barrier. Curr Opin Nephrol Hypertens 2009;18:331-5.

4. Patrakka J, Tryggvason K. New insights into the role of podocytes in proteinuria. Nat Rev Nephrol 2009;5:463-8.

5. Eremina V, Sood M, Haigh J, Nagy A, Lajoie G, Ferrara N, et al. Glomerular-specific alterations of VEGF-A expression lead to distinct congenital and acquired renal diseases. J Clin Invest 2003;111:707-16

6. Banas MC, Banas B, Hudkins KL, Wietecha TA, Iyoda M, Bock E, et al. TLR4 links podocytes with the innate immune system to mediate glomerular injury. J Am Soc Nephrol 2008;19:704-13.

7. Reiser J, von Gersdorff G, Loos M, Oh J, Asanuma K, Giardino L, et al. Induction of B7-1 in podocytes is associated with nephrotic syndrome. J Clin Invest 2004;113:1390-7.

8. Trimarchi H. Abatacept in glomerular diseases. The open road for the second signal as a new target is settled down. Recent Patents on Endocrine, Metabolic \& Immune Drug Discovery 2015;9:1-13.

9. Luyckx VA, Brenner BM. The clinical importance of nephron mass J Am Soc Nephrol 2010;21:898-910.

10. Tan JC, Workeneh B, Busque S, Blouch K, Derby G, Myers BD. Glomerular function, structure, and number in renal allografts from older deceased donors. J Am Soc Nephrol 2009;20:181-8.

11. Romagnani P, Lasagni L, Remuzzi G. Renal progenitors: An evolutionary conserved strategy for kidney regeneration. Nat Rev Nephrol 2013;9:137-46.

12. Vogelmann SU, Nelson WJ, Myers BD, Lemley KV. Urinary excretion of viable podocytes in health and renal disease. Am J Physiol Renal Physiol 2003;285:F40-8.

13. Sagrinati C, Netti GS, Mazzinghi B, Lazzeri E, Liotta F, Frosali F, et al. Isolation and characterization of multipotent progenitor cells from 
the Bowman's capsule of adult human kidneys. J Am Soc Nephrol 2006;17:2443-56.

14. Mazzinghi B, Ronconi E, Lazzeri E, Sagrinati C, Ballerini L, Angelotti ML, et al. Essential but differential role for CXCR4 and CXCR7 in the therapeutic homing of human renal progenitor cells. J Exp Med 2008;205:479-90.

15. Ronconi, E, Sagrinati C, Angelotti ML, Lazzeri E, Mazzinghi B, Ballerini L, et al. Regeneration of glomerular podocytes by human renal progenitors. J Am Soc Nephrol 2009;20:322-32.

16. Lazzeri E, Crescioli C, Ronconi E, Mazzinghi B, Sagrinati C, Netti GS, et al. Regenerative potential of embryonic renal multipotent progenitors in acute renal failure. J Am Soc Nephrol 2007;18:3128-38.

17. Angelotti ML, Ronconi E, Ballerini L, Peired A, Mazzinghi B, Sagrinati $C$, et al. Characterization of renal progenitors committed toward the tubular lineage and their regenerative potential in renal tubular injury. Stem Cells 2012;30:1714-25.

18. Sallustio F, De Benedictis L, Castellano G, Zaza G, Loverre A, Costantino V, et al. TLR2 plays a role in the activation of human resident renal stem/progenitor cells. FASEB J 2010;24:514-25.

19. Kriz W, Shirato I, Nagata M, LeHir M, Lemley KV. The podocyte's response to stress: The enigma of foot process effacement. Am J Physiol Renal Physiol 2013;304:F333-47.

20. Couser WG. Basic and translational concepts of immune-mediated glomerular diseases J Am Soc Nephrol 2012;23:381-99.

21. Trimarchi H. Primary focal and segmental glomerulosclerosis and suPAR: Where do we stand and where are we heading to?. World J Nephrol 2013;2:103-22.

22. Wei C, Moller CC, Altintas MM, Li J, Schwarz K, Zacchigna S, et al. Modification of kidney barrier function by the urokinase receptor. Nat Med 2008;14:55-63.

23. Jefferson JA, Shankland SJ, Pichler RH. Proteinuria in diabetic kidney disease: A mechanistic viewpoint. Kidney Int 2008;74:22-36.

24. Shankland SJ. The podocyte's response to injury: Role in proteinuria and glomerulosclerosis. Kidney Int 2006;69:2131-47.

25. Wharram BL, Goyal M, Wiggins JE, Sanden SK, Hussain S, Filipiak WE, et al. Podocyte depletion causes glomerulosclerosis: Diphtheria toxin-induced podocyte depletion in rats expressing human diphtheria toxin receptor transgene. J Am Soc Nephrol 2005;16:2941-52.

26. Peti-Peterdi J, Sipos A. A high-powered view of the filtration barrier. J Am Soc Nephrol 2010;21:1835-41.

27. Becker JU, Hoerning A, Schmid KW, Hoyer PF. Immigrating progenitor cells contribute to human podocyte turnover. Kidney Int 2007;72:1468-73.

28. Ohse T, Vaughan MR, Kopp JB, Krofft RD, Marshall CB, Chang AM, et al. De novo expression of podocyte proteins in parietal epithelial cells during experimental glomerular disease. Am J Physiol Renal Physiol 2010;298:F702-11.

29. Kriz W. The pathogenesis of ' classic' focal segmental glomerulosclerosislessons from rat models. Nephrol Dial Transplant 2003;18 (Suppl 6):vi39-44.

30. Hara M, Yamamoto T, Yanagihara T, Takada T, Itoh M, Adachi Y, et al. Urinary excretion of podocalyxin indicates glomerular epithelial cell injuries in glomerulonephritis. Nephron 1995;69:397-403.

31. Hara $M$, Yanagihara $T$, Itoh $M$, Matsuno $M$, Kihara I. Immunohistochemical and urinary markers of podocyte injury. Pediatr Nephrol 1998;12:43-8.

32. Nakamura T, Ushiyama C, Suzuki S, Hara M, Shimada N, Ebihara I, et al. Urinary excretion of podocytes in patients with diabetic nephropathy. Nephrol Dial Transplant 2000;15:1379-83.
33. Nakamura T, Ushiyama C, Suzuki S, Hara M, Shimada N, Sekizuka $\mathrm{K}$, et al. Urinary podocytes for the assessment of disease activity in lupus nephritis. Am J Med Sci 2000;320:112-6.

34. Nakamura T, Ushiyama C, Suzuki S. Effect of angioten-sin-converting enzyme inhibitor, angiotensin II receptor antagonist and calcium antagonist on urinary podocytes in patients with IgA nephropathy. Am J Nephrol 2000;20:373-9.

35. Sekulic M, Pichler-Sekulic S. A compendium of urinary biomarkers indicative of glomerular podocytopathy. Patholog Res Int 2013; 2013:782395.

36. Wickman L, Afshinnia F, Wang SQ, Yang Y, Wang F, Chowdhury M, et al. Urine podocyte mRNAs, proteinuria, and progression in human glomerular diseases. J Am Soc Nephrol 2013;24:2081-95.

37. Hara M, Yanagihara T, Kihara I. Significance of urinary binucleated podocytes in IgA nephropathy. Nephrology 2001;6:A15.

38. Ponchiardi C, Fall B, Scott R, Uhrich S, Mauer M, Whitley C, et al. Podocyturia correlates with proteinuria in patients with Fabry disease (FD) and is a potential biomarker of Fabry nephropathy. Mol Genet Metab 2013;108:S76-7.

39. Ryu M, Mulay S, Misoge N, Gross O, Anders H. Tumor necrosis factor- $\beta$ drives Alport glomerulosclerosis in mice by promoting podocyte apoptosis. J Pathol 2012;226:120-31.

40. Endlich N, Kress K, Reiser J, Uttenweiler D, Kriz W, Mundel P, et al. Podocytes respond to mechanical stress in vitro. J Am Soc Nephrol 2001;12:413-22.

41. Grishman E, Churg J. Focal glomerular sclerosis in nephrotic patients: An electron microscopic study of glomerular podocytes. Kidney Int 1975;7:111-22.

42. Hara M, Yanagihara T, Hirayama Y, Ogasawara S, Kurosawa H, Sekine $\mathrm{S}$, et al. Podocyte membrane vesicles in urine originate from tip vesiculation of podocyte microvilli. Hum Pathol 2010;41:1265-75.

43. Böttinger E. TGF-beta in renal injury and disease. Semin Nephrol 2007;3:309-20.

44. Maestroni S, Maestroni A, Dell'Antonio G, Gabellini D, Terzi S, Spinello A, et al. Viable podocyturia in healthy individuals: Implications for podocytopathies. Am J Kidney Dis 2014;64:1003-5.

45. Jopling C, Boue S, Izpisua Belmonte JC. Dedifferentiation, transdifferentiation and reprogramming: Three routes to regeneration. Nat Rev Mol Cell Biol 2011;12:79-89.

46. Herman-Edelstein M, Thomas MC, Thallas-Bonke V, Saleem M, Cooper ME, Kantharidis P. Dedifferentiation of immortalized human podocytes in response to transforming growth factor-b: A model for diabetic podocytopathy. Diabetes 2011;60:1779-88.

47. Meyer TW, Bennett PH, Nelson RG. Podocyte number predicts longterm urinary albumin excretion in Pima Indians with Type II diabetes and microalbuminuria. Diabetologia 1999;42:1341-4.

48. Steffes MW, Schmidt D, McCrery R, Basgen JM. Glomerular cell number in normal subjects and in type 1 diabetic patients. Kidney Int 2001;59:2104-13.

49. Trimarchi H, Muryan A, Raña MS, Paggi P, Lombi F, Forrester M, et al. Proteinuria and its relation to diverse biomarkers and body mass index in chronic hemodialysis. Int J Nephrol Renovasc Dis 2013;6:113-9.

How to cite this article: Trimarchi H. Podocyturia: What is in a name? J TransI Intern Med 2015;3:51-56

Source of Support: NIL, Conflict of Interest: NIL 